\title{
Plasma Metabolomic Profiling in Workers With Noise- induced Hearing Loss: A Pilot Study
}

\section{Long Miao ( $\square$ miaolong0308@163.com )}

Southeast University https://orcid.org/0000-0001-6949-5855

\section{Boshen Wang}

Southeast University

Juan Zhang

Southeast University

\section{Lihong Yin}

Southeast University

\section{Yuepu Pu}

Southeast University

\section{Research Article}

Keywords: Noise-induced hearing loss, Metabolomics, Plasma, Gene, Autophagy

Posted Date: May 10th, 2021

DOI: https://doi.org/10.21203/rs.3.rs-437429/v1

License: (1) (i) This work is licensed under a Creative Commons Attribution 4.0 International License. Read Full License

Version of Record: A version of this preprint was published at Environmental Science and Pollution Research on July 17th, 2021. See the published version at https://doi.org/10.1007/s11356-021-15468-z. 


\section{Abstract}

Noise-induced hearing loss $(\mathrm{NIHL})$ remains a leading occupational related disease and is a serious public health problem. Hence, the identification of potential biomarkers for NIHL prevention and diagnosis has become an urgent work. To discover potential metabolic biomarkers of NIHL, plasma metabolomics analysis among 62 NIHL patients and 62 controls was performed using ultrahigh performance liquid chromatography-mass spectrometry (UPLC/MS). Orthogonal partial least square-discriminant analysis (OPLS-DA) model was applied to distinguish metabolite profile alterations in plasma samples between the two groups. The alterations in autophagy pathway were in accordance with previous published studies, therefore, three autophagy-related genes (PI3K, AKT and ATG5) were selected and mRNA levels were detected by RT-qPCR analysis in peripheral white blood cells (WBCs) samples. Compared to the control group, 20 identified plasma metabolites were significantly altered in NIHL patients. Meanwhile, a total of seven metabolic pathways were enriched, including glycerophospholipid metabolism, glycosylphosphatidylinositol (GPI)-anchor biosynthesis, autophagy, choline metabolism, alpha-linolenic acid metabolism and linoleic acid metabolism, and retrograde endocannabinoid signaling pathway. Furthermore, the results indicated that the mRNA levels of three autophagy-related genes (PI3K, AKT and ATG5) were significantly decreased in NIHL cases compared with controls. Taken together, our current study firstly provides evidence that the identified aberrantly altered metabolites might be potential biomarkers of NIHL for noise-exposed workers. In addition, autophagy pathway may be involved in the occurrence and development of NIHL.

\section{Introduction}

Occupational noise, a common harmful factor that seriously affects the health of workers in the field of occupational health. Noise-induced hearing loss $(\mathrm{NIHL})$ is one of the worst adverse health effect induced by occupational noise exposure in workplaces (Masterson et al. 2016). The World Health Organization (WHO) has reported that about ten percent of the world's population is exposed to high level of noise and is at risk of developing $\mathrm{NIHL}$ (Basner et al. 2014). Nowadays, NIHL is an urgent health problem and has a strong impact on social economy and human health. According to previous study, $\mathrm{NIHL}$ is one of the leading occupational related disease in China, accounting for approximately one-sixth of the annual increase of occupational disease (Miao et al. 2019). Although a number of studies were conducted, the specific pathogenesis underlying NIHL has still not been entirely illustrated. Therefore, it is of great significance to search new and potential biomarkers and further explore the mechanism underlying NIHL.

Metabolites in human body fluids represent the end products of metabolic pathways and reflect the final consequences of organisms in response to environmental stimulation and disease stress (Huang et al. 2018). More importantly, these endogenous metabolic changes can indicate the direct biological response to internal and external stressors, such as environmental exposure, disease and nutritional imbalances (Wang et al. 2017). Now, metabolomics has become a powerful and effective platform to identify extensive biomolecule closely correlated with environmental factors and health effect (Chen et al. 2019, Huang et al. 2018). Thus, identification of metabolic biomarkers could help to better understand the possible molecular mechanism of adverse health effects induced by harmful exposure factors and may further contribute to high-risk individuals' identification (Floegel et al. 2013).

It has been reported that a key factor contributing to NIHL is oxidative stress damage to body's sensory hair cells (Yuan et al. 2015). Oxidative stress is a state of imbalance between oxidation and antioxidant defenses, thus resulting in oxidative damage (Sies 1997). Available studies showed that reactive oxygen species (ROS) may play an essential role in regulating cellular stress and defense pathways. Excessive production of ROS was thought to be a 
key pathological mechanism involving in the process of inner ear injury, such as exposure to noise and ototoxic drug therapy (Ohlemiller et al. 1999, Yamashita et al. 2004). In addition, related studies found that ROS has the capacity to induce cell defense pathways like autophagy. Autophagy is an important defense process that could pass impaired cell components to lysosomes degradation (Wang \&Klionsky 2003, Yang \&Klionsky 2009). In our previous studies, we found that inflammation-related genes polymorphisms associated with the susceptibility to NIHL, revealing inflammation is an essential stress to the pathogenesis of noise-induced cochlear impairment (Miao et al. 2021a, Miao et al. 2021b). Nevertheless, the related metabolic profiles in occupational noise-exposed workers are still not clear and whether autophagy is involved in the development of NIHL has yet to be established and need to be further explored.

In this study, metabolomics of plasma samples from occupational noise-exposed workers with hearing loss and normal hearing controls was performed to identify potential metabolic biomarkers and pathways participating in the process of NIHL development. We further conducted quantitative PCR reaction to determine the mRNA expression levels of a few crucial genes in autophagy pathway.

\section{Materials And Methods}

\section{Subjects}

The study subjects were the workers who exposed to occupational noise in the factories. According to requests, all workers were requested to receive annual occupational health examination, such as general physical examination, peripheral blood collection and pure-tone audiometry (PTA) test. The questionnaire survey was performed to collect important information of subjects, including general family history of disease, personal disease history, smoking and alcohol consumption, and drug use history. In this study, the criteria for inclusion of study subjects were: (1) workers who exposed to occupational noise for more than one year; (2) workers only exposed to occupational noise; (3) Chinese Han workers. Nevertheless, the exclusion criteria were as follows: (1) workers who had family history of deafness; (2) workers who carried diseases that could affect normal hearing (e.g., otitis media, tinnitus, and skull trauma); (3) workers had recently treatment with ototoxic drugs (e.g., aminoglycosides, quinolones, aspirin) that damages the normal function of the inner ear; (4) workers had metabolic diseases (e.g., diabetes, hypertension, hyperlipidemia, etc.).

This study was approved by the Ethics Committee of Zhongda Hospital Affiliated to Southeast University and written informed consent was also acquired from all study subjects.

\section{Noise exposure evaluation}

Exposure level of noise was estimated according to equivalent continuous $\mathrm{dB}(\mathrm{A})$-weighted sound pressure levels ( $\mathrm{L}_{\text {Aeq }} 8 \mathrm{~h}$ ) with the TES 1350A sound level meter (TES, Taiwan). Cumulative noise exposure (CNE) was used to reflect the true individual noise exposure level based on $L_{\text {Aeq. }}$.

\section{Pure-tone audiometric examination}

After stopping noise exposure for over $12 \mathrm{~h}$, each study subject has to accept the PTA examination conducted by professional doctor using Voyager 522 audiometer (Madsen, Taastrup, Denmark) at frequencies of 0.5, 1, 2, 3, 4, and $6 \mathrm{kHz}$ in a noise-proof room. Referring to GB/T7582-2004, the obtained raw data were adjusted by sex and age.

\section{Selection of NIHL cases and normal hearing controls}


Referring to the Chinese National Occupational Health Standard (GBZ49-2007), NIHL cases of this current study were define as occupational noise-exposed workers with binaural high frequencies $(3,4 \mathrm{and} 6 \mathrm{kHz})$ hearing threshold level more than $25 \mathrm{~dB}(\mathrm{~A})$. Nevertheless, those with binaural high frequencies hearing threshold level less than $25 \mathrm{~dB}$ (A) were included as the normal hearing controls. Totally, 124 subjects including $62 \mathrm{NIHL}$ cases and 62 normal hearing controls were recruited in this study.

\section{Chemicals and Reagents}

Liquid chromatography grade methanol and acetonitrile were purchased from Merck (Darmstadt, Germany). Both formic acid and ammonium formate were purchased from Thermo Fisher Scientific (Waltham, USA). Ultrapure water was prepared by Milli-Q water purify system (Millipore, USA).

\section{Plasma sample collection and preparation}

Considering food intake and drinking may result in the alteration of human metabolome, morning peripheral blood (collected after $12 \mathrm{~h}$-fasting) is collected for plasma collection. Two milliliters of peripheral venous blood were collected from each study subject and transferred to tubes containing ethylenediaminetetraacetic acid. Plasma samples were isolated and centrifuged at $3500 \mathrm{rpm}$ at room temperature for $15 \mathrm{~min}$. Subsequently, all plasma samples were stored at $-80^{\circ} \mathrm{C}$ until metabolomics analysis.

All collected plasma samples were thawed at $4{ }^{\circ} \mathrm{C}$ and then vortexed for $20 \mathrm{~s}$. A $100 \mu \mathrm{L}$ aliquot of plasma sample was mixed using three times volume of methanol for protein precipitation. Then, the mixture was further vortexed for $30 \mathrm{~s}$ and was centrifuged at $13000 \mathrm{rpm}$ at $4{ }^{\circ} \mathrm{C}$ for $20 \mathrm{~min}$. The liquid supernatant was obtained for centrifugation at $13000 \mathrm{rpm}$ at $4^{\circ} \mathrm{C}$ for $20 \mathrm{~min}$. Finally, a total of $20 \mu \mathrm{L}$ aliquot of the supernatant was transferred into a sample vial for metabolomics analysis.

\section{Chromatographic and mass spectrometric analysis}

Metabonomic profiles of plasma samples from occupational noise-exposed workers was performed by ACQUITY Ultra Performance Liquid Chromatography (UPLC) (Waters, Milford, USA) system equipped with AB SCIEX Triple TOF 5600 System (AB SCIEX, Framingham, USA).

Chromatographic separation was implemented on an ACQUITY UPLC BEH C8 column $(2.1 \mathrm{~mm} \times 100 \mathrm{~mm} \times 1.7 \mu \mathrm{m}$, Waters, Milford, USA). For positive ion mode, the mobile phase was composed of water with $0.1 \%$ formic acid (A) and acetonitrile (B). For negative ion mode, the mobile phase was $5 \mathrm{mM}$ ammonium formate aqueous solution (C) and acetonitrile (D). The procedures of gradient elution were as follows: $5 \%$ solution $B$ for $0-0.5$ min, $5-20 \%$ solution B for $0.5-2$ min, $20-25 \%$ solution B for $2-4$ min, $25-60 \%$ solution B for $4-10$ min, and $60-100 \%$ solution B for $10-15$ min, $100 \%$ solution $B$ for $15-16 \mathrm{~min}$, and $5 \%$ solution $B$ for $16-19 \mathrm{~min}$. The delivery flow rate was set at $0.3 \mathrm{~mL} / \mathrm{min}$. Besides, the injection volume was $5 \mu \mathrm{L}$. All analyzed samples were kept at $4{ }^{\circ} \mathrm{C}$, and the temperature of column was set at $40{ }^{\circ} \mathrm{C}$.

Mass spectrometry (MS) was conducted with AB SCIEX Triple TOF 5600 System (AB SCIEX, Framingham, USA) coupled with electrospray ionization source. The parameters were as follows: collision energy, $35 \mathrm{~V} /-35 \mathrm{~V}$; declustering potential, $80 \mathrm{~V} /-80 \mathrm{~V}$; ion spray voltage, $5000 \mathrm{~V} /-4500 \mathrm{~V}$; pressure of nebulizer gas, 50 psi; and curtain gas, 35 psi. Besides, the interface heater temperature was $500^{\circ} \mathrm{C}$ and $550{ }^{\circ} \mathrm{C}$, respectively. The scanning range of data acquisition was $50-1200 \mathrm{~m} / \mathrm{z}$.

\section{Data processing and analysis}


Firstly, the original data were processed using Progenesis QI software for baseline removing, peak identification, peak alignment and integration, and retention time adjustment. Then, a data matrix composed of retention time, mass-to-charge ratio, intensity of peak and information of sample was performed for further statistical analyses. For each plasma sample, the peak intensity corresponding to metabolite was further adjusted with the total intensity of peak of sample.

SIMCA-P 14.1 (Umetrics, Umea, Sweden) was applied for multivariate statistical analyses. Both principal component analysis (PCA) and orthogonal partial least square-discriminant analysis (OPLS-DA) were applied for distinguishing normal hearing controls and NIHL patients after unit variance (UV) scaling. The metabolites with variable importance of projection (VIP) values > 1 calculated by SIMCA-P were considered to be the potential biomarkers. Furthermore, a heatmap of metabolites was generated with Multi Experiment Viewer (MEV) software.

Some further analyses were also conducted in this study. MetaboAnalyst was applied for related metabolic pathway analysis (http://www.metaboanalyst.ca). Pathway plots were based on the Kyoto Encyclopedia of Genes and Genomes (KEGG) database. Gene Set Enrichment Analysis Software (GSEA, Broad Institute, Cambridge, MA, USA) was used to gene enrichment analysis. Furthermore, to characterize the gene functions, Human metabolome database (HMDB) was performed.

\section{RNA extraction and RT-qPCR analysis}

TRIZOL reagent (Invitrogen, Carlsbad, CA) was employed for RNA extraction. Double-stranded cDNA was synthesized with Takara Prime Script RT Reagent Kit (Takara Bio Inc., Clontech, Japan) referring to the instructions.

Subsequently, RT-qPCR was conducted with SYBR Green real-time PCR kits (Toyobo, Osaka, Japan). The following primers were used for mRNA expression levels detection: PI3K, forward: 5'-CCACGACCATCATCAGGTGAA-3', reverse: 5'-CCTCACGGAGGCATTCTAAAGT-3'; AKT, forward: 5'-GCAAGGTGATCCTGGTGAA-3', reverse: 5'TCGTGGGTCTGGAAAGAGTA-3'; ATG5, forward: 5'-AAAGATGTGCTTCGAGATGTGT-3', reverse: 5'CACTTTGTCAGTTACCAACGTCA-3'; and $\beta$-actin, forward: 5'-CTACCTCATGAAGATCCTCACCGA-3', reverse: 5'TTCTCCTTAATGTCACGCACGATT-3'.

\section{Statistical analyses}

Statistical analysis was carried out to ensure the potential metabolites significantly changed between NIHL cases and controls by using paired non-parametric test on MATLAB software (MathWorks). Otherwise, statistical analysis was conducted with the SPSS 23.0 software (SPSS, Chicago, Illinois, USA). The statistical significance criterion was set with a two-sided $P$ value $<0.05$.

\section{Results}

\section{Characteristics of subjects}

A total of 62 NIHL cases and 62 healthy controls were recruited in this study. In terms of age, sex, time with noise exposure, habit of smoking and drinking, systolic blood pressure (SBP) and diastolic blood pressure (DBP) levels, and exposure level with noise, no significant differences were found between the case group and control group ( $P>$ 0.05). Nevertheless, a significant difference of the high frequency hearing threshold were observed between two groups. The results showed that NIHL patients had a significantly higher hearing threshold of high frequency in both ears (53.24 \pm 13.01$)$ compared with the controls $(18.73 \pm 3.96 ; P<0.001)$. The information of all study subjects are summarized in Table 1. 


\section{Plasma metabonomic profiling of NIHL}

The non-targeted metabolomic profile was explored in 124 plasma samples obtained from 62 NIHL patients and 62 controls using UPLC-Q-TOF/MS with ESI positive ion mode and negative ion mode. Due to the metabolites detected by positive ion mode and negative ion mode were complementary, we combined the data obtained from two modes into a matrix for analysis. After removing missing values $>50 \%$ ion peaks, 6777 ion peaks in positive ion mode and 5404 ion peaks in negative ion mode were obtained. A total of 4009 metabolites (2277 in positive ion mode and 1732 in negative ion mode) were detected by accurate mass, fragmentation patterns and retention time. Univariate statistical analyses were further performed for all metabolite to determine the changes of plasma metabolome between two groups. Based on OPLS-DA model, an obviously separation was shown in NIHL patients and normal hearing (Figure 1).

\section{Identification of changed endogenous metabolites}

Paired non-parametric test was conducted to identify significant differential metabolites for plasma between two groups. Totally, 207 differential metabolites $(P<0.05)$ were identified, including 136 up-regulated and 71 downregulated metabolites. We further explored which significant differential metabolites contributing to the differences described above by setting comparatively low stringency $(P<0.05$ and VIP $>1)$. In this way, it showed that 59 metabolites were significantly alterations in NIHL patients compared with the controls. Through further examining the original data and feature of ion peaks, 20 metabolites were identified and considered potential biomarkers, including 12 up-regulated and 8 down-regulated metabolites (Table 2). Besides, a visual heat map was generated based on the 20 plasma differential metabolites, showing a considerable difference between NIHL and controls (Fig. 2). The up-regulated metabolites were organic acids, including homodeoxycholic acid, quinolacetic acid, etc. Nevertheless, most of down-regulated metabolites were lipids, such as glycerophospholipids and saccharolipids, etc. KEGG pathway analysis showed that seven metabolic pathways were enriched, including glycerophospholipid metabolism, glycosylphosphatidylinositol (GPI)-anchor biosynthesis, autophagy, choline metabolism, alpha-linolenic acid metabolism and linoleic acid metabolism, and retrograde endocannabinoid signaling pathway, suggesting these pathways may be involved in the development of NIHL (Table 3).

\section{Autophagy related-gene expression in NIHL patients and controls}

Among the identified differentially regulated metabolites, $\mathrm{PE}(15: 0 / 20: 2(11 Z, 14 Z))$, also known as Phosphatidylethanolamine in HMDB, was found to be reduced in plasma samples in NIHL patients, which was involved in autophagy metabolism pathway. In addition, the another metabolite, namely, PC(15:0/18:1(11Z)) (called Phosphatidylcholine in HMDB) was also decreased in plasma samples of NIHL patients and was involved in autophagy metabolism pathway. To explore the biological effects of noise exposure on autophagy pathway in NIHL patients, three autophagy-related genes (PI3K, AKT, and ATG5) were included and the expression levels were determined in peripheral white blood cells (WBCs) of NIHL patients and controls. Results indicated that individuals with NIHL had significantly lower levels of PI3K, AKT, and ATG5 than those with normal hearing (Fig. 3).

\section{Discussion}

$\mathrm{NIHL}$ is one of the serious harmful health effects induced by high-intensity noise exposure and has become a leading occupation-related disease in the world (Miao et al. 2019). In the present study, we compared the plasma metabolomic characteristics of NIHL patients with normal hearing controls by using non-targeted metabolomics, which provides a theoretical basis for further exploring the pathogenesis of harmful effects induced by noise. Totally, 
twenty significantly changed metabolites were identified, revealing disturbances of a variety of biological pathways in the development of NIHL. We performed three autophagy related-gene expression levels by conducting RT-qPCR and found that PI3K, AKT, and ATG5 were significantly downregulated in NIHL patients compared with controls, suggesting that autophagy pathway plays an essential role in development of NIHL. Therefore, our study firstly provides a new perspective to understand the mechanism and identifies potential biomarkers closely correlated with $\mathrm{NIHL}$ and verified the critical role of autophagy pathway in NIHL.

Metabolomics is a powerful platform for exploring disease phenotype, which provides a wealth of information for the discovery of biomarker, pathogenesis and personalized treatment (Ye et al. 2014). So far, some metabolomics studies have been performed in noise exposure field (Floegel et al. 2013, Huang et al. 2018, Wang et al. 2017, Zhang et al. 2016). Pudrith et al. (Pudrith \&Dudley 2019) found five metabolites related to glutathione-dependent mercapturic acids in urine, while significant associations were just only found in non-noise exposure subjects. Fujita et al. (Fujita et al. 2015) revealed that ten metabolites exhibited statistically significant changes in inner ear fluid of guinea pig that exposed to loud noise, including amino acid catabolites and lipid compounds. Also, a study by He et al. (He et al. 2017)demonstrated that multiple metabolic pathways are involved in acoustic trauma, such as arginine, proline, and purine metabolic pathways. However, little studies were conducted to investigate the metabolic signatures induced by occupational noise in humans. Thus, we analyzed the plasma samples of $62 \mathrm{NIHL}$ patients and 62 normal hearing controls to explore the metabolomic profiles on NIHL. Eventually, 20 differential metabolites previously unknown were identified to be correlated with NIHL.

Bilirubin is one of important products of heme catabolism and is an effective antioxidant that removes hydrogen peroxide (Minetti et al. 1998). Bilirubin and glutathione were reported to have complementary cell-protective and antioxidant effects (Sedlak et al. 2009). Findings from previous studies indicated that serum bilirubin was closely related to cardiovascular disease-related factors, including body mass index, metabolic syndrome, and diabetes (Cheriyath et al. 2010, Horsfall et al. 2012). In addition, studies found that the plasma and serum bilirubin level significantly increased in benzene-exposed workers compared with the controls (Neghab et al. 2015). In our current study, low plasma bilirubin in NIHL patients may act in conjunction with glutathione to protect cells from oxidation. However, the concrete role of bilirubin in the development of NIHL needs to be further explored.

Among the identified differential metabolites, some were phospholipids (PLs), which are major components of cellular membranes and vital bioactive molecules (Cvetkovic et al. 2017). In this study, plasma levels of a few of crucial PLs (PE(15:0/20:2(11Z,14Z)), PC(15:0/18:1(11Z)), and PI(0-20:0/18:0)) were significantly decreased in NIHL patients compared with controls. It is well known that lipid metabolism is frequently occurred in a variety of diseases, but the most recent evidence found that lipid-related genes may be involved in inflammatory and metabolic diseases (Hirsch et al. 2010). Previous studies have found that the alterations of PLs levels in tissue or plasma may be associated with the risk and progression of all kinds of diseases (Sun et al. 2018). Cvetkovic et al. (Cvetkovic et al. 2017) had reported that the abnormal alterations of PLs profile were associated with non-Hodgkin's lymphoma. In addition, lipidomic studies suggested that the altered levels of PLs composition could results in changes in membrane integrity, permeability, cell damage, as well as cell intimal transport (Leamy et al. 2014). Metabolites PE, $\mathrm{PC}$ and $\mathrm{PI}$, also known as phosphatidylethanolamine, phosphatidylcholine and phosphatidylinositol, a group of antioxidants, were involved in cell morphology, metabolism regulation, signal transduction, and a variety of physiological functions of cells (Hidalgo et al. 2005). In this study, the decreased levels of three metabolites in plasma of NIHL patients may be due to the overproduction of ROS during noise exposure, thereby being consumed to maintain the balance between ROS and antioxidant defenses system. Meanwhile, this finding may indicate that oxidative stress is a key factor and important mechanism contributing to NIHL. 
In addition, the results obtained from our study showed that the abnormal metabolites, both PE and PC were involved in autophagy pathway, indicating autophagy may be closely associated with the development of NIHL. Recent findings demonstrated that PE is an important substrate for the GPI-anchor biosynthesis that is essential for immune response and plays an essential role in the initiation of autophagy by attaching to the autophagy protein to initiate autophagosome formation (Fracchiolla et al. 2017). The findings from previous studies indicated that ROS have the capacity to induce cell defense pathways like autophagy, which is a protective process that delivers negative cellular components to lysosomes for degradation (Wang \&Klionsky 2003, Yang \&Klionsky 2009). Abnormal level of metabolites in autophagy pathway was observed in NIHL patients, indicating that autophagy may play a potential biological role in NIHL. Therefore, we conducted RT-qPCR to explore the mRNA expression of important genes in autophagy metabolism pathway. PI3K/AKT pathway is an upstream major modulator of autophagy and they participate in extensive cellular process, including cell growth, proliferation, survival and metabolism (Heras-Sandoval et al. 2014). ATG5, as an essential autophagy related protein, is involved in autophagy at the molecular level (Zheng et al. 2019). Taken together, these three genes were used for further validation. Our results displayed that the mRNA levels of PI3K, AKT and ATG5 were decreased in the WBC of NIHL patients. Substantial evidence has suggested that the reduction of PI3K/AKT signaling pathways is correlated with hair cell death and hearing loss after various of injuries and stimuli (Chen et al. 2015). Data from animal studies showed that deletion of ATG5 could cause hair cells degeneration and profound congenital hearing loss in mice (Fujimoto et al. 2017). In addition, the most data from a review showed that autophagy is a key factor for the auditory cell fate, but autophagy deficiency may be one of leading causes of hearing impairment (Hayashi et al. 2020). Our results are in accord with the previously reported findings, indicating that autophagy could be considered an essential signaling pathway involved in NIHL development.

\section{Conclusions}

In summary, our study firstly provides evidence that metabolomics can characterize the metabolites and provide novel insights into the molecular events triggered by NIHL. We found that the plasma metabonomic profiles significantly altered in NIHL patients compared with normal hearing controls. Twenty identified metabolites were significantly altered in NIHL patients. The metabolic signature alterations of metabolites indicated that some specific pathways were involved in NIHL, including

glycosylphosphatidylinositol (GPI)-anchor biosynthesis, autophagy, choline metabolism, alpha-linolenic acid metabolism and linoleic acid metabolism, retrograde endocannabinoid signaling, and glycerophospholipid metabolism. These altered metabolites might act as potential biomarkers of NIHL diagnosis for Chinese noiseexposed workers. In addition, autophagy pathway might play an essential role in the occurrence and development of $\mathrm{NIHL}$. This study provides a new perspective for the progress of $\mathrm{NIHL}$ and offers novel clues on the mechanisms underlying NIHL.

\section{Declarations}

\section{Acknowledgments}

The authors thank every worker for their participations in this study.

\section{Authors' contributions}


YP designed the research study. LM and BW performed the research and analyzed the data. LM drafted the manuscript. JZ, LY critically reviewed and revised the manuscript. All authors read and approved the final manuscript.

\section{Funding}

This study was supported by the Open Research Fund of State Key Laboratory of Bioelectronics, Southeast University.

\section{Data availability}

The datasets used and analyzed during the current study are available from the corresponding author on reasonable request.

\section{Compliance with ethical standards}

\section{Conflict of interest}

The authors declare that they have no conflicts of interest.

\section{Ethical approval}

The present study was approved by the Ethics Committee of Zhongda Hospital, Affiliated Hospital of Southeast University.

\section{Consent to participate}

Informed consent was obtained from all individual participants included in the study.

\section{Consent to publish}

The participant has consented to the submission of the case report to the journal.

\section{References}

1. Basner M, Babisch W, Davis A, Brink M, Clark C, Janssen S, Stansfeld S (2014) Auditory and non-auditory effects of noise on health. Lancet 383:1325-1332

2. Chen C, Li H, Niu Y, Liu C, Lin Z, Cai J, Li W, Ge W, Chen R, Kan H (2019) Impact of short-term exposure to fine particulate matter air pollution on urinary metabolome: A randomized, double-blind, crossover trial. Environ Int 130:104878

3. Chen J, Yuan H, Talaska AE, Hill K, Sha SH (2015) Increased Sensitivity to Noise-Induced Hearing Loss by Blockade of Endogenous PI3K/Akt Signaling. J Assoc Res Otolaryngol 16:347-356

4. Cheriyath P, Gorrepati VS, Peters I, Nookala V, Murphy ME, Srouji N, Fischman D (2010) High Total Bilirubin as a Protective Factor for Diabetes Mellitus: An Analysis of NHANES Data From 1999 - 2006. J Clin Med Res 2:201206

5. Cvetkovic Z, Milosevic M, Cvetkovic B, Masnikosa R, Arsic A, Petrovic S, Vucic V (2017) Plasma phospholipid changes are associated with response to chemotherapy in non-Hodgkin lymphoma patients. Leuk Res 54:39-46 
6. Floegel A, Stefan N, Yu Z, Muhlenbruch K, Drogan D, Joost HG, Fritsche A, Haring HU, Hrabe de Angelis M, Peters A, Roden M, Prehn C, Wang-Sattler R, Illig T, Schulze MB, Adamski J, Boeing H, Pischon T (2013) Identification of serum metabolites associated with risk of type 2 diabetes using a targeted metabolomic approach. Diabetes 62:639-648

7. Fracchiolla D, Zens B, Martens S (2017) In Vitro Reconstitution of Atg8 Conjugation and Deconjugation. Methods Enzymol 587:377-390

8. Fujimoto C, Iwasaki S, Urata S, Morishita H, Sakamaki Y, Fujioka M, Kondo K, Mizushima N, Yamasoba T (2017) Autophagy is essential for hearing in mice. Cell Death Dis 8:e2780

9. Fujita T, Yamashita D, Irino Y, Kitamoto J, Fukuda Y, Inokuchi G, Hasegawa S, Otsuki N, Yoshida M, Nibu K (2015) Metabolomic profiling in inner ear fluid by gas chromatography/mass spectrometry in guinea pig cochlea. Neurosci Lett 606:188-193

10. Hayashi K, Suzuki Y, Fujimoto C, Kanzaki S (2020) Molecular Mechanisms and Biological Functions of Autophagy for Genetics of Hearing Impairment. Genes (Basel) 11

11. He J, Zhu Y, Aa J, Smith PF, De Ridder D, Wang G, Zheng Y (2017) Brain Metabolic Changes in Rats following Acoustic Trauma. Front Neurosci 11:148

12. Heras-Sandoval D, Perez-Rojas JM, Hernandez-Damian J, Pedraza-Chaverri J (2014) The role of $\mathrm{PI3K} / \mathrm{AKT} / \mathrm{mTOR}$ pathway in the modulation of autophagy and the clearance of protein aggregates in neurodegeneration. Cell Signal 26:2694-2701

13. Hidalgo FJ, Nogales F, Zamora R (2005) Changes produced in the antioxidative activity of phospholipids as a consequence of their oxidation. J Agric Food Chem 53:659-562

14. Hirsch HA, lliopoulos D, Joshi A, Zhang Y, Jaeger SA, Bulyk M, Tsichlis PN, Shirley Liu X, Struhl K (2010) A transcriptional signature and common gene networks link cancer with lipid metabolism and diverse human diseases. Cancer Cell 17:348-361

15. Horsfall LJ, Nazareth I, Petersen I (2012) Cardiovascular events as a function of serum bilirubin levels in a large, statin-treated cohort. Circulation 126:2556-2564

16. Huang Q, Hu D, Wang X, Chen Y, Wu Y, Pan L, Li H, Zhang J, Deng F, Guo X, Shen H (2018) The modification of indoor PM2.5 exposure to chronic obstructive pulmonary disease in Chinese elderly people: A meet-in-metabolite analysis. Environ Int 121:1243-1252

17. Leamy AK, Egnatchik RA, Shiota M, Ivanova PT, Myers DS, Brown HA, Young JD (2014) Enhanced synthesis of saturated phospholipids is associated with ER stress and lipotoxicity in palmitate treated hepatic cells. J Lipid Res 55:1478-1488

18. Masterson EA, Bushnell PT, Themann CL, Morata TC (2016) Hearing Impairment Among Noise-Exposed Workers - United States, 2003-2012. MMWR Morb Mortal Wkly Rep 65:389-394

19. Miao L, Ji J, Wan L, Zhang J, Yin L, Pu Y (2019) An overview of research trends and genetic polymorphisms for noise-induced hearing loss from 2009 to 2018. Environ Sci Pollut Res Int 26:34754-34774

20. Miao L, Wang B, Ji J, Wan L, Yin L, Zhu B, Zhang J, Pu Y (2021a) CARD8 polymorphism rs2043211 protects against noise-induced hearing loss by causing the dysfunction of CARD8 protein. Environ Sci Pollut Res Int 28:8626-8636

21. Miao L, Wang B, Zhang J, Yin L, Pu Y (2021b) A functional SNP in miR-625-5p binding site of AKT2 3'UTR is associated with noise-induced hearing loss susceptibility in the Chinese population. Environ Sci Pollut Res Int

22. Minetti M, Mallozzi C, Di Stasi AM, Pietraforte D (1998) Bilirubin is an effective antioxidant of peroxynitritemediated protein oxidation in human blood plasma. Arch Biochem Biophys 352: 165-174 
23. Neghab M, Hosseinzadeh K, Hassanzadeh J (2015) Early Liver and Kidney Dysfunction Associated with Occupational Exposure to Sub-Threshold Limit Value Levels of Benzene, Toluene, and Xylenes in Unleaded Petrol. Saf Health Work 6:312-316

24. Ohlemiller KK, Wright JS, Dugan LL (1999) Early elevation of cochlear reactive oxygen species following noise exposure. Audiol Neurootol 4:229-236

25. Pudrith C, Dudley WN (2019) Sensorineural hearing loss and volatile organic compound metabolites in urine. Am J Otolaryngol 40:409-412

26. Sedlak TW, Saleh M, Higginson DS, Paul BD, Juluri KR, Snyder SH (2009) Bilirubin and glutathione have complementary antioxidant and cytoprotective roles. Proc Natl Acad Sci U S A 106:5171-5176

27. Sies H (1997) Oxidative stress: oxidants and antioxidants. Exp Physiol 82:291-295

28. Sun R, Xu K, Zhang Q, Jiang X, Man Z, Yin L, Zhang J, Pu Y (2018) Plasma metabonomics investigation reveals involvement of fatty acid oxidation in hematotoxicity in Chinese benzene-exposed workers with low white blood cell count. Environ Sci Pollut Res Int 25: 32506-32514

29. Wang CW, Klionsky DJ (2003) The molecular mechanism of autophagy. Mol Med 9:65-76

30. Wang X, Liu L, Zhang W, Zhang J, Du X, Huang Q, Tian M, Shen H (2017) Serum metabolome biomarkers associate low-level environmental perfluorinated compound exposure with oxidative /nitrosative stress in humans. Environ Pollut 229:168-176

31. Yamashita D, Jiang HY, Schacht J, Miller JM (2004) Delayed production of free radicals following noise exposure. Brain Res 1019:201-209

32. Yang Z, Klionsky DJ (2009) An overview of the molecular mechanism of autophagy. Curr Top Microbiol Immunol 335:1-32

33. Ye G, Liu Y, Yin P, Zeng Z, Huang Q, Kong H, Lu X, Zhong L, Zhang Z, Xu G (2014) Study of induction chemotherapy efficacy in oral squamous cell carcinoma using pseudotargeted metabolomics. J Proteome Res 13:1994-2004

34. Yuan H, Wang X, Hill K, Chen J, Lemasters J, Yang SM, Sha SH (2015) Autophagy attenuates noise-induced hearing loss by reducing oxidative stress. Antioxid Redox Signal 22:1308-1324

35. Zhang J, Liu L, Wang X, Huang Q, Tian M, Shen H (2016) Low-Level Environmental Phthalate Exposure Associates with Urine Metabolome Alteration in a Chinese Male Cohort. Environ Sci Technol 50:5953-5960

36. Zheng W, Xie W, Yin D, Luo R, Liu M, Guo F (2019) ATG5 and ATG7 induced autophagy interplays with UPR via PERK signaling. Cell Commun Signal 17:42

\section{Tables}


Table 1 Demographic characteristics of NIHL patients and control subjects

\begin{tabular}{lllll} 
Characteristic & \multicolumn{2}{c}{ Cases $(\mathrm{n}=62)$} & Controls $(\mathrm{n}=62)$ & $P$ \\
\cline { 2 - 4 } & $\mathrm{n}$ & $\%$ & $\mathrm{n}$ & $\%$
\end{tabular}

Age (years)

$0.832^{b}$

Mean \pm SD

$42.11 \pm 10.15$

$41.73 \pm 10.07$

Sex

Male

Female

62

100.0

62

100.0

Work time with noise (years)

0.0

0

0.0

Mean \pm SD

$18.19 \pm 11.94$

$18.32 \pm 11.71$

Smoking status

$\begin{array}{llll}0 & 0.0 & 0 & 0.0\end{array}$

$P$
n

0.832

$\begin{array}{lllll}\text { No } & 18 & 29.0 & 19 & 30.6 \\ \text { Yes } & 44 & 71.0 & 43 & 69.4\end{array}$

Drinking status

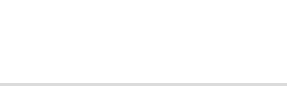

No
Yes
SBP

29

46.8

25

40.3

33

53.2

37

59.7

SBP

Mean \pm SD

$124.42 \pm 17.03$

$126.15 \pm 15.38$

DBP

Mean \pm SD

$80.27 \pm 11.35$

$81.18 \pm 13.00$

High frequency hearing threshold (dB)

Mean \pm SD

$53.24 \pm 13.01$

$18.73 \pm 3.96$

Expose level with noise (dB)

$0.971^{b}$

Mean \pm SD

$89.11 \pm 3.84$

$89.08 \pm 5.86$

a Two-sided $\chi^{2}$ test.

b Students' t-test.

SD, standard deviation; dB, decibel; SBP, systolic blood pressure; DBP, diastolic blood pressure 
Table 2 Identified plasma differential metabolites associated with NIHL

\begin{tabular}{|c|c|c|}
\hline $\begin{array}{l}\text { Accuracy } \\
\text { mass (m/z) }\end{array}$ & $\begin{array}{l}\text { Retention } \\
\text { time (min) }\end{array}$ & Metabolites \\
\hline
\end{tabular}

Positive ion

mode

\begin{tabular}{|c|c|c|c|c|c|}
\hline 311.1852569 & 10.4648 & Botrydial & 1.27286759 & 0.03894814 & 1.28783125 \\
\hline 185.0443214 & 6.67985 & 3-0-Methylgallate & 2.31339463 & 0.02983198 & 1.70865564 \\
\hline 459.2490377 & 8.34475 & Homodeoxycholic acid & 1.18948582 & 0.01139502 & 1.53923459 \\
\hline 169.049315 & 8.169933333 & Quinolacetic acid & 3.29015824 & 0.03374699 & 1.70844877 \\
\hline 239.1643354 & 12.32853333 & $\begin{array}{l}\text { 7-oxo-11E,13-Tetradecadienoic } \\
\text { acid }\end{array}$ & 1.57982426 & 0.03663327 & 1.33969105 \\
\hline 237.1482017 & 10.25615 & $\begin{array}{l}\text { 12,13-Dimethyl-5,14- } \\
\text { dioxabicyclo[9.2.1]-tetradeca- } \\
\text { 1(13),11-dien-4-one }\end{array}$ & 1.04849816 & 0.00652026 & 1.45013866 \\
\hline 540.3658642 & 12.40703333 & PS(0-20:0/0:0) & 1.06692078 & 0.03151553 & 0.84758463 \\
\hline 167.033526 & 5.928883333 & 3,4-Dihydroxymandelic acid & 1.7126814 & 0.03719973 & 1.51393039 \\
\hline 708.4889779 & 12.71418333 & $\begin{array}{l}\text { Butyl 4'-0-butanoyl-6-0- } \\
\text { hexadecanoyl-neohesperidoside }\end{array}$ & 2.0716998 & 0.03969699 & 0.56778489 \\
\hline 752.5186207 & 12.68268333 & $\operatorname{PE}(15: 0 / 20: 2(11 Z, 14 Z))$ & 2.14374406 & 0.0216304 & 0.60668356 \\
\hline 313.0358286 & 0.7735 & $\begin{array}{l}\text { 4-Hydroxy-5-(3'-hydroxyphenyl)- } \\
\text { valeric acid-3'-0-sulphate }\end{array}$ & 1.49168798 & 0.0243405 & 1.14578276 \\
\hline 838.2387531 & 7.86895 & $\begin{array}{l}\text { (\{2-[2-(2-hydroxypropan-2-yl)-7- } \\
\text { oxo-2H,3H,7H-furo[3,2- } \\
\text { g]chromen-6-yl]-2-methylbut-3- } \\
\text { en-1-yl\}oxy) sulfonic acid }\end{array}$ & 3.61397217 & 0.02653202 & 2.32513639 \\
\hline 837.4362666 & 7.86895 & Mabioside D & 3.93298292 & 0.02712122 & 2.37605848 \\
\hline 763.5942952 & 11.72606667 & $P C(15: 0 / 18: 1(11 Z))$ & 1.41579684 & 0.01678653 & 0.40772182 \\
\hline 616.2519865 & 9.662016667 & 12S-acetoxy-punaglandin 1 & 1.02041589 & 0.04217161 & 0.70668393 \\
\hline 881.6498 & 8.8424 & $\mathrm{PI}(0-20: 0 / 18: 0)$ & 1.60990954 & 0.03281649 & 0.74938231 \\
\hline
\end{tabular}

Negative ion

mode

\begin{tabular}{|llllll|}
\hline 583.2554212 & 7.870583333 & Bilirubin & 5.94802089 & 0.01894616 & 0.75876997 \\
\hline 245.0484576 & 7.240283333 & $\begin{array}{l}\text { [3-(4- } \\
\text { methoxyphenyl)propoxy]sulfonic } \\
\text { acid }\end{array}$ & 1.77471751 & 0.02071462 & 2.1502221 \\
\hline 615.244282 & 7.947083333 & $\begin{array}{l}\text { Myricanene B 5-[arabinosyl-(1- } \\
>6 \text { )-glucoside] }\end{array}$ & 1.74800988 & 0.02618671 & 0.74123558 \\
\hline 311.29438 & 16.92686667 & Stearic Acid ethyl ester & 1.16524736 & 0.00478247 & 1.62621185 \\
\hline
\end{tabular}

VIP, variable importance of projection; FC, fold change 


\begin{tabular}{|lll|}
\hline \multicolumn{2}{|l|}{ Table 3 Identified differential metabolic pathways involved in NIHL } & \\
\hline Pathway name & P-value & FDR correction \\
\hline Retrograde endocannabinoid signaling & $1.51 \mathrm{E}-04$ & $2.27 \mathrm{E}-03$ \\
\hline Glycerophospholipid metabolism & $1.16 \mathrm{E}-03$ & $5.79 \mathrm{E}-03$ \\
\hline Glycosylphosphatidylinositol (GPI)-anchor biosynthesis & $3.26 \mathrm{E}-03$ & $9.79 \mathrm{E}-03$ \\
\hline Autophagy & $4.35 \mathrm{E}-03$ & $1.09 \mathrm{E}-02$ \\
\hline Choline metabolism & $1.19 \mathrm{E}-02$ & $2.24 \mathrm{E}-02$ \\
\hline Linoleic acid metabolism & $3.01 \mathrm{E}-02$ & $5.02 \mathrm{E}-02$ \\
\hline Alpha-Linolenic acid metabolism & $4.71 \mathrm{E}-02$ & $6.42 \mathrm{E}-02$ \\
\hline FDR, false discovery rate & & \\
\hline
\end{tabular}

\section{Figures}

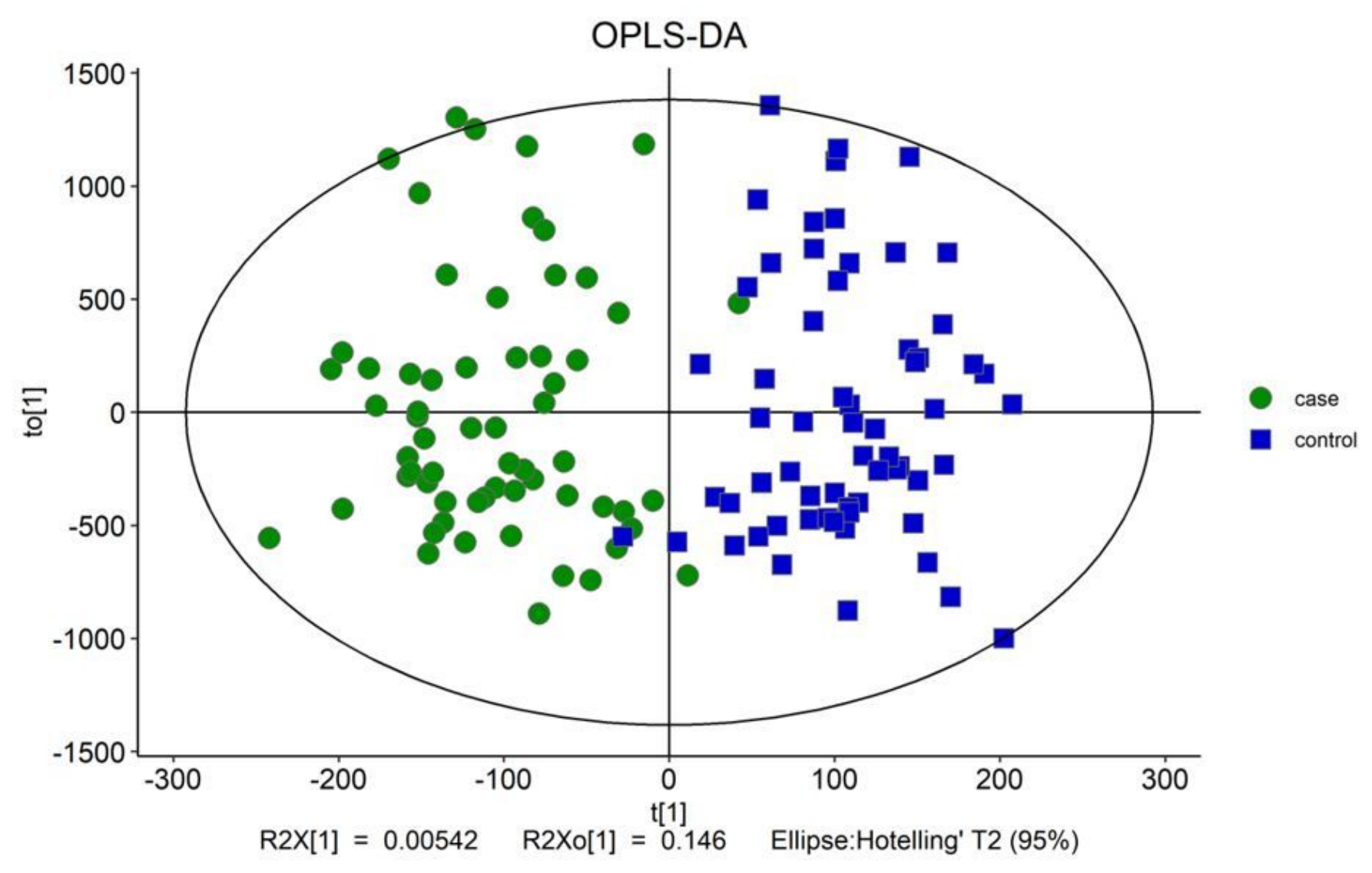


Figure 1

Scoring plots for OPLS-DA model. OPLS-DA score plot of healthy control group (blue) and NIHL group (green). Data from positive ion mode and negative ion mode were combined.

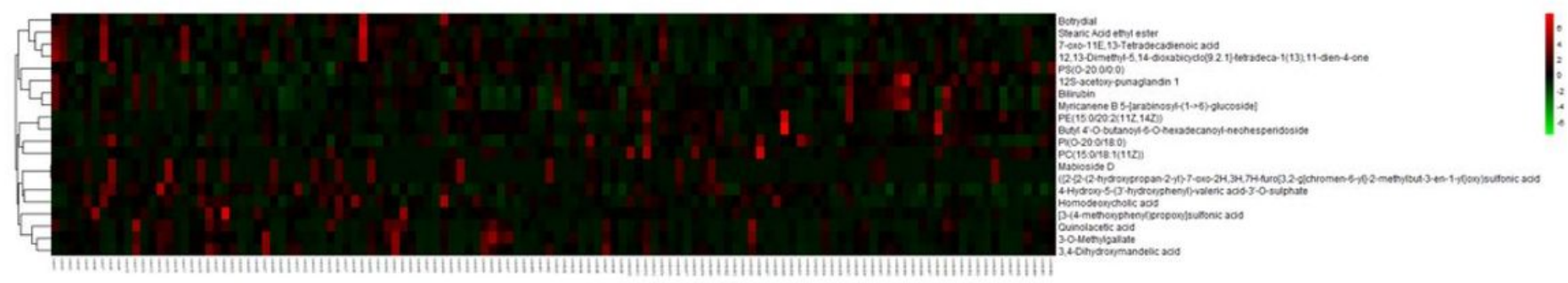

\section{Figure 2}

Heat map of twenty significantly changed metabolites. Red color indicates a high level of metabolites and green color indicates a low level of metabolites, while black color means an equal level in both groups.

a

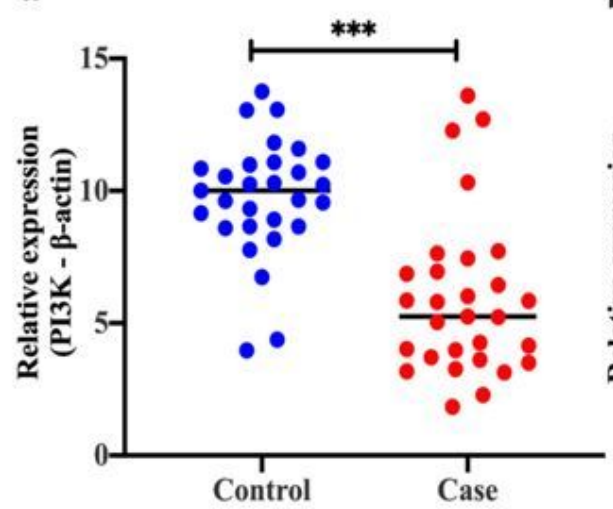

b

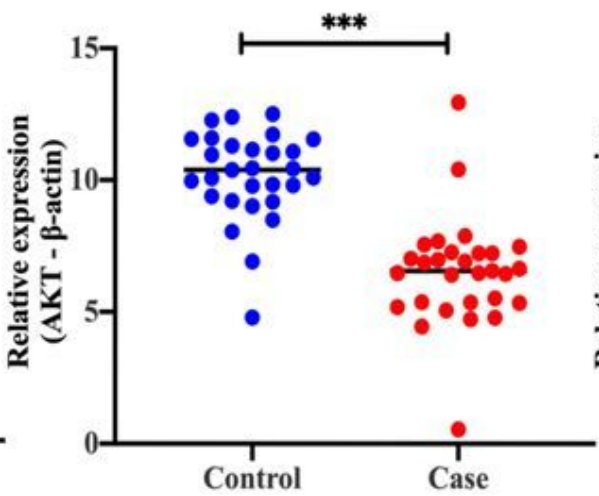

c

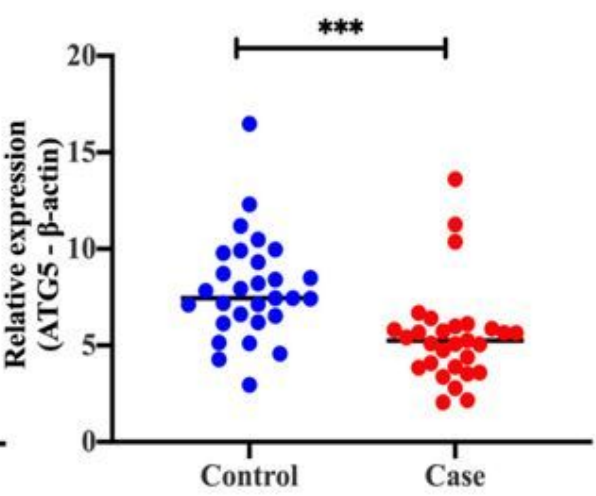

Figure 3

mRNA levels of PI3K, AKT and ATG5 in NIHL patients and controls were detected by RT-qPCR analysis. ${ }^{\star * \star P}<0.001$. 